\title{
Vivre de sa fiction, vivre dans sa fiction : le processus créateur au défi du numérique
}

\author{
Aimie Shaw \\ Université de Washington \\ États-Unis
}

L'auteur est de retour dans son récit, sa mort n'a pas été accomplie : il est une chimère, un caméléon, un fantôme qui hante nos lectures. II prétend parfois garder ses distances de son récit, mais il porte le même prénom que son protagoniste. Ce dernier déclare même écrire des romans. Un pur hasard? Peut-être. Mais, en même temps, le protagoniste fait souvent référence aux ouvrages antérieurs de l'auteur, à ses entretiens et aussi à son blog quotidien. C'est bien sûr une ruse : on peut poser l'hypothèse que l'auteur se projette dans le texte afin de se confondre avec son personnage. II s'immisce dans son texte pour y déclarer son autorité et pour narrer son récit. Pour ainsi dire, il résiste à sa propre mort, telle qu'annoncée par Roland Barthes en $1967^{1}$, et reprend tout contrôle sur l'intentionnalité du texte. Mais, subitement, intervient une autre voix, par le biais d'une digression ou d'une note de bas de page, déclarant que c'est lui l'auteur et qu'il faut, de toute évidence, éviter la confusion naïve entre lui et son protagoniste. Tel est le ton, la tension-et peut-être même la prétention-qui marquent l'œuvre du romancier contemporain Éric Chevillard.

La distinction entre fiction et réalité soulignée ci-dessus est appuyée par Roland Barthes lorsque dans «Introduction à l'analyse structurale du récit », paru en 1966, il envisage pour la première fois des " niveaux du récit »(6). Puisque le récit est considéré comme « objet », il " est l'enjeu d'une communication : il y a un donateur du récit, il y a une destinataire du récit. [...] [I]l ne peut y avoir de récit sans narrateur et sans auditeur (ou lecteur) » (18-19). Une distinction entre représentation et référentialité est désormais établie par la binarité entre réalité et fiction qui soustend les niveaux de Barthes, ce dernier appuyant également que « narrateur et personnages sont essentiellement des "êtres de papier" ; l'auteur (matériel) d'un récit ne peut se confondre en rien avec le narrateur de récit » (19). Le monde romanesque, ou plus largement fictionnel, est à considérer comme étant tout à fait autonome, indépendant de la réalité, ce qui garantit l'homogénéité des figures qui y résident. Cette distinction a également pour conséquence l'effacement de l'auteur-personne et son intentionnalité de l'interprétation, réduisant l'auteur à une fonction de la création. Le schéma narratologique ${ }^{2}$ ainsi que les théories de la réception ${ }^{3}$ qui découlent de ces premières analyses structuralistes deviennent la lentille à travers laquelle nous examinons un objet littéraire, inspirant pourtant un langage conceptuel qui devient un instigateur actif d'un discours sur les possibles du roman et d'une réflexion sur la tension narrative créée entre le langage fictionnel et l'interprétation réelle.

Cette visée théorique est défiée sur le plan esthétique chez plusieurs auteurs contemporains, qui, par le biais de la fausse sincérité et de brouillages intentionnels s'insèrent dans leurs récits, forgeant un discours métafictionnel. Dans les ouvrages d'Éric Chevillard, Christian Gailly, Christine Angot et Ying Chen, exemples parmi tant d'autres, l'écrivain ou l'écrivaine prend délibérément une posture ambivalente, menant à une extension réelle de l'esthétique de sa fiction. Bien que la présence de l'auteur ne soit pas nouvelle en soi, la posture d'écrivain-personnage dans le roman contemporain sert désormais à interroger le statut du roman et le processus créateur. II est caractérisé d'une autoréflexivité qui remet en cause la notion même de représentation, la possibilité d'une autorité auctoriale et la nature arbitraire de la division binaire entre fiction et réalité. Loin d'être un stratagème pour vanter son pouvoir, il s'agit d'un auteur descendu de son socle, créant ainsi un « anti-mythe de l'écrivain » (Držková 73): un " statut officieux fait de proximité, d'échange et de dialogues, et non plus celui que confère une parole autorisée et prestigieuse » (Viart et Vercier 301). Que ce soit de nouveaux rapports dialogiques forgés entre auteur et lecteur, entre auteur et personnage ou, de façon plus large, entre réalité et fiction, le mythe transformé en anti-mythe annonce un auteur protéiforme et omniprésent, agissant non pas comme autorité mais comme collaborateur prêt à négocier le texte avec ses lecteurs et personnages.

Or, à l'ambiguïté entre auteur-personne, auteur-narrateur ou écrivain-personnage qui découle de l'anti-mythe de l'auteur, s'ajoute désormais une autre catégorie, à savoir l'« auteur-numérique ». L'éclatement de la littérature, par sa forme aussi bien que par sa quantité, initié par la publication en ligne des textes, mène à une préoccupation pointue sur notre capacité, ou surtout sur notre incapacité, de juger celle-ci. Nous sommes vite enclins à nous prononcer sur l'état des choses et à rejeter le travail " moins sérieux " des écrivains du numérique, qui succombent aux facilités naïves de notre époque ou aux désirs du lecteur de consommation rapide, proposant avec un certain dégoût que tout lecteur est désormais créateur. D'une part, nous faisons allusion à la limite de 140 caractères sur Twitter, à la formule de profil sur Facebook ou aux contraintes arbitraires imposées par les écrivains eux-mêmes sur leur blog quotidien. D'autre part, nous évoquons une liberté trop large une fois le livre débarrassé des contraintes du genre, des éditeurs et des librairies. La remise en question des catégories théoriques auxquelles nous nous sommes habitués pour saisir et juger les rapports entre auteur, lecteur et personnages laisse la critique à la croisée de la tradition et de l'expérimentation avec peu de repères. Et pourtant, le texte numérique ne semble pas être une mode passagère. 
Le livre n'adhère guère à son image uniquement matérielle. Sommes-nous donc, comme le propose François Bon, " après le livre »? Nous sommes, du moins, à une époque où le texte matériel est potentiellement court-circuité, corrompu ou compliqué par le texte numérique. La question n'est pourtant pas binaire. Nombreux sont les écrivains, comme François Bon, avec un pied dans les deux mondes : des auteurs fidèles à une maison d'édition, tout comme au maintien de leur site-web. Au-delà d'une machine purement promotionnelle, les auteurs dédoublent l'image d'euxmêmes qui est " convertie en profil-composite inédit d'actes, d'intentions et d'indices [...] pour questionner ce qu'elle était censée exposer : notre identité » (Merzeau 30). Dans la littérature, cet éclatement identitaire avance toutefois un projet d'écrivain singulier, même si hétérogène et transmédiatique. II s'agit, comme le suggère Alexandre Gefen dans "Le devenir numérique de la littérature française », d'une extension des théories deleuziennes (le nomadisme identitaire) et barthésiennes (le sujet " scriptible »), auxquelles nous pouvons ajouter celles de Marshall McLuhan (les médias "chauds » et «froids ") et de Nicolas Bourriaud (l'esthétique relationnelle). Nous sommes dans la continuité de la rupture : le virtuel vient compléter le matériel.

Dans son projet d'écrivain, Éric Chevillard aborde plusieurs ruptures : celles de la division nette entre fiction et réalité, de l'opposition entre auteur et narrateur, et désormais de notre compréhension des figures de personne et de persona. Auteur d'une vingtaine de romans publiés majoritairement aux Éditions de Minuit, il accorde régulièrement des entretiens, presque jamais en personne, surtout par courriel, dont le thème récurrent est le processus créateur de son propre œuvre expérimentale mise en dialogue avec une critique poussée du manque d'imagination d'autres écrivains de son époque et la paresse du lecteur contemporain. II s'approprie l'outil informatique afin de mobiliser une présence virtuelle qui renforce l'ambiguïté entre auteur-personne et auteur-narrateur et qui façonne son œuvre analogue. Or, sa présence virtuelle ou numérique à travers son site web et son blog quotidien en particulier, confirme un désir de maintenir une ambiguïté entre personne et persona, ce qui est renforcé par le fait qu'il se permet de s'insérer directement en tant qu'auteur-personne dans ses fictions et de se mêler aux diverses figures d'écrivainspersonnages qui peuplent ses romans. Comme Chevillard, ces écrivains-personnages prennent pour objet le récit qui s'écrit, la littérature au sens plus large et l'activité créatrice.

La réflexion qui parcourt toute son œuvre et commente le statut de la littérature contemporaine en dévoilant son processus de réalisation est la plus évidente dans les nombreuses notes en bas de page qui complètent son roman, L'auteur et moi, paru en 2012. Le titre complique déjà l'identité auctoriale : qui est l'« auteur " en question, qui est ce « moi » et quel est leur rapport à l'autre nom qui figure sur la page de couverture, à savoir « Éric Chevillard »?

À travers cette étude de cas nous mettons en dialogue la présence virtuelle de l'auteur dans le péritexte, notamment ses entretiens, son site-web et son blog, avec la critique à la fois implicite et explicite du paratexte de L'auteur et moi, à savoir la quatrième de couverture, l'avertissement au lecteur et les notes en bas de page. Ce faisant, nous soulignons à la fois l'impact de la confluence virtuelle, fictionnelle et réelle sur son projet d'écrivain, et aussi, selon Chevillard, une autre manière de concevoir le processus créateur et le genre romanesque.

Nous avançons l'hypothèse que la présence virtuelle de l'auteur devient une extension de cette tension initiée entre auteur et narrateur. De plus, cette multiplication des brouillages défait la binarité des rapports entre fiction et réalité de façon à créer une souplesse esthétique et théorique parmi les diverses figures qui résident traditionnellement dans ces deux sphères. En ce qui concerne plus particulièrement cette étude de cas, la présence numérique de l'auteur renforce et avance le projet d'écrivain de Chevillard, à savoir celui de " [s]'attaque[r] à la littérature. Elle est l'espace dans lequel depuis toujours s'inscrit [s]a propre existence » («Des leurres » 14). II s'agit d'un projet transmédiatique qui complète le métadiscours au cœur de l'œuvre chevillardienne. Cette réflexion sera inspirée du récit principal de L'auteur et moi, qui raconte l'histoire d'un « je » anonyme dans un café avec une femme. II commande une truite aux amandes, mais reçoit un gratin de chou-fleur, « la plus injustifiable, la plus déprimante, la plus nuisible [...] des inventions humaines » (255). L'intrigue tourne presque exclusivement autour du gratin de choufleur, qui devient une véritable obsession pour le protagoniste. Ce dernier réussit à manger ce repas qui le dégoûte, tout en réfléchissant à sa passion plus raffinée pour la truite aux amandes. Au cœur de cette considération il y a un commentaire plus large sur la littérature d'aujourd'hui et les figures de l'auteur, du personnage et du lecteur.

\section{Qui est le chef cuisinier?}

La déclaration audacieuse qui se présente sur la page de couverture en tant que titre du roman donne le ton tendu qui se développera par la suite entre le narrateur du récit principal et celui des notes en bas de page. La quatrième de couverture de L'auteur et moi anticipe le questionnement du lecteur en s'interrogeant si ce livre est :

[...] un récit humoristique délirant, une confession autobiographique désarmante, un essai polémique agressif, ou bien plutôt, outrepassant ces catégories qui se télescopent ici, tantôt joyeusement, tantôt brutalement, une mise à l'épreuve de la vie de l'auteur dans le champ de la littérature où il s'est établi au saut du berceau ? (Quatrième de couverture) 
Le lecteur de Chevillard est habitué à ce type de confluence esthétique et formelle qui marque son œuvre et ne s'attend pas à une réponse dans le récit. Sa complexité sera plutôt explorée, sinon exploitée, en jouant sur les attentes du lecteur vis-à-vis du genre romanesque. L'interrogation de la quatrième de couverture souligne la confusion créée dans cet ouvrage entre le langage référentiel et le langage fictionnel. On lui accorde une place privilégiée par le biais d'une réflexion qui porte sur l'identité de l'auteur et du protagoniste. À la première page de l'avertissement, nous lisons :

Les récits de l'auteur affectent le plus souvent une forme vaguement romanesque et il doit composer de ce fait avec quelques-uns au moins des principes de réalité du roman et, par exemple, dans certains cas, nommer ses personnages. C'est une première épreuve pour son imagination stérile qui se révèle totalement inapte à cela, incapable de créer ex nihilo un patronyme vraisemblable et cependant euphonique et original. Ainsi l'auteur a-t-il couramment recours à l'usurpation d'identité ou, plus modestement, car il ne s'agit en somme que de faire usage d'un nom sans prétendre dépouiller qui que ce soit de ses qualités et prérogatives, à l'emprunt. Et, afin de ne contrarier personne ni susciter de fâcheuse confusion, il prélève de préférence ces noms sur les tombes. (7-8)

Critiquant d'abord le manque d'imagination de l'auteur, l'avertissement souligne ensuite que la construction romanesque des personnages commence par un emprunt à la réalité. La fausse promesse de maintenir une distinction nette entre " êtres réels » et " êtres fictifs » est d'abord renforcée par la mise en page du texte matériel. Les quarante notes en bas de page qui y figurent séparent textuellement le récit fictionnel et son narrateur des commentaires référentiels des notes. Selon l'auteur de l'avertissement, celles-ci serviront à l'auteur du roman " d'intervenir en personne à chaque fois que les circonstances l'exigeront, avec sang-froid et fermeté, pour se garder de toute confusion » (16). La promesse matérielle d'ontologies distinctes et de mondes possibles homogènes semble pour un moment se tenir lorsque le lecteur est confronté pour la première fois au « je » anonyme du récit principal et est renvoyé directement à une première note où la voix narrative tente de dissiper toute confusion, selon laquelle ce « je » ressemble à l'auteur du récit (19-20). La voix narrative des notes devient en quelque sorte le porte-parole d'Éric Chevillard et soutient l'auteur dans sa démarche de distanciation. Cet effort est très développé : les notes en bas de page occupent une place importante dans le récit, non seulement par le rôle qu'elles jouent dans la distinction du monde réel et des mondes possibles de la fiction, mais surtout par leur ampleur. Elles s'étendent parfois sur plusieurs pages et en plus d'éclaircir ou d'ajouter de façon pertinente au texte, elles tendent davantage vers les digressions alambiquées auxquelles l'œuvre chevillardienne nous a habitués. De plus, du point de vue typographique, la police et la taille des caractères des notes sont semblables aux caractères du texte principal. Or, la première note déclare l'envie de l'auteur de "prendre le dessus et de dominer son sujet » (19) et l'avertissement annonce sa conviction " à se tenir à distance du narrateur de son nouveau récit, à se démarquer de lui aussi clairement que possible afin de rester maître de la situation »(16). Toutefois, dans une parenthèse du récit principal figure malgré tout une description d'une douzaine de lignes, qui, d'après la note 20 , « se retrouve textuellement dans le journal de l'auteur à la date du 17 décembre $2010 »(90)$. Si l'auteur déclare garder ses distances par rapport à son narrateur, il brouille simultanément le rapport entre les deux, suggérant qu'il reconnaît la naïveté dans tout propos qui cherche à extraire la voix réelle de l'auteur de la voix fictionnelle du narrateur. Dans la note 8 , il commente en effet le rôle que joue le langage dans la construction de la et de la fiction :

Il lui paraît qu'en laissant s'emballer la logique du discours qui fonde notre réalité entièrement inventée par le langage [...] [l'auteur] mettra au jour l'imposture que constitue celle-ci, puisque justement elle n'est qu'un fait ou un effet de langue. Seule la mort se passe de mots-serait-elle la seule réalité ; et tout le reste une fiction dont nous serions à la fois les auteurs et les personnages ? (47)

En interrogeant la valeur d'authenticité attribuée à la réalité, Chevillard jette un défi aux frontières délicates qui la constitue. II témoigne d'une confusion inévitable entre la voix auctoriale et la voix narrative par une mise en pratique de cette démarcation futile non seulement dans les notes de ce roman mais aussi sur son blog, sur son site-web et dans ses entretiens.

La figure auctoriale tripartite proposée par Dominique Maingueneau dans Le discours littéraire : paratopie et scène d'énonciation est propice à l'appréhension de la tension dialogique forgée par la crise identitaire auctoriale. La réflexion de Maingueneau invite à nuancer la binarité identitaire fort connue et répandue entre "personne » et « écrivain » par le biais d'une troisième dimension, l'« inscripteur » (107-108). Si les deux premières instances évoquent la distinction entre "vie privée » et "vie publique », l'inscripteur est "à la fois énonciateur d'un texte particulier et, qu'il le veuille ou non, le ministre de l'Institution littéraire qui donne sens aux contrats impliqués par les scènes génériques et s'en porte garant » (107-108). L'inscripteur est donc celui qui se situe au sein d'un texte afin de raconter une histoire tout en respectant les attentes extratextuelles du genre, qui lui imposent les possibilités d'énonciation et qui reflètent éventuellement les attentes éditoriales et interprétatives. Maingueneau refuse ainsi 
d'établir une frontière étanche entre le texte et le hors-texte en insistant sur une porosité nécessaire à l'appréhension de l'inscripteur. D'ailleurs, comme il précise :

Ces trois instances ne se disposent pas en séquence, que ce soit en termes de chronologie ou de strates. [...] [C]hacune des trois instances est traversée par les deux autres, aucune n'est fondement ou pivot. [...] Chacune des trois soutient les deux autres et se soutient d'elles dans un processus d'enveloppement réciproque qui, d'un même mouvement, disperse et rassemble « le » créateur. (108)

Les trois instances sont entrelacées, faisant de l'inscripteur une figure multiple et protéiforme. Or, la notion d'inscripteur n'est pas strictement analogue et s'étend aussi au domaine du numérique, prenant tout son sens dans le cadre de l'œuvre chevillardienne. L'auteur-personne, caractérisé par sa vie privée se confond avec l'inscripteur lorsque paraît sur le site-web professionnel d'Éric Chevillard sa "biographie », qui est toutefois écrite par l'auteur même, semant ainsi le doute sur son authenticité. Qui plus est, la biographie se lit comme n'importe quelle description ludique de ses protagonistes :

Éric Chevillard, né un 18 juin à la Roche-sur-Yon, anciennement Napoléon-Vendée, il ne s'endort pas pour autant sur ses lauriers puisqu'on le voit encore effectuer bravement ses premiers pas cours Cambronne, à Nantes. II a deux ans lorsqu'il met un terme à sa carrière de héros national. II brise alors son sabre sur son genou puis raconte à sa mère qu'il s'est écorché en tombant de cette balançoire et elle feint gentiment de le croire.

Ensuite, il écrit. Purs morceaux de délire selon certains, ses livres sont pourtant l'œuvre d'un logicien fanatique. L'humour est la conséquence imprévue de ses rigoureux travaux.

II partage son temps entre la France (trente-neuf années) et le Mali (cinq semaines). Hier encore, un de ses biographes est mort d'ennui. (Chevillard, « Biographie »)

La vie privée s'étend à cet espace public et sa biographie dans l'espace virtuel remet en question la distinction entre auteur et inscripteur. II est également à noter que le texte date de 2004 : il est donc une version de Chevillard qui n'est plus lui, mais une trace écrite de lui, rapprochant l'auteur d'une figure textuelle d'un univers fictionnel.

L'inscripteur, comme le souligne Ugo Ruiz dans «Ethos et blog d'écrivain : le cas de L'Autofictif d'Éric Chevillard », joue aussi un rôle important dans le cadre du blog personnel que l'écrivain tient à jour depuis 2007. Ruiz note que :

Chevillard entend imposer l'inscripteur par l'usage d'une "parlure » qui renvoie à des phénomènes linguistiques dans l'appréhension littéraire du monde. [...] Le ton ironique généralement utilisé définit une « posture d'énonciation » par laquelle Chevillard crée des effets comiques et multiplie les niveaux d'interprétation possibles installant l'énonciation dans un métadiscours qui a pour objet l'écriture ellemême $[\ldots]$.

Dans son blog, Chevillard rapproche divers tons et thèmes. Par exemple, le 19 septembre 2014, il écrit :

En septembre 1987, ton grand-père fêta ses 50 ans et je publiai mon premier livre. À mon tour cette année déjà d'atteindre cet âge. Et toi, Agathe [sa fille], tu apprends seulement à lire... ! II va falloir se remuer un peu, ma belle ! (L'Autofictif)

À ce moment intime entre lui et sa fille il mêle des actualités littéraires : « [p]armi les jurés du Prix Renaudot, Frédéric Beigbeder, Patrick Besson et Franz-Olivier Giesbert-c'est dire l'insigne honneur qui échoit chaque année aux non lauréats " (L'Autofictif). De surcroît, il écrit des récits fictionnels sur les personnages récurrents dans ses textes matériels-comme Crab et Albert Moindre ${ }^{4}$-en plus de récits introduisant de nouveaux personnages. Le blog ébranle l'homogénéisation générique, se servant approximativement de la contrainte de publier trois textes quotidiennement.

Enfin, cette même présence d'inscripteur-auteur s'étend aux entretiens où Chevillard déclare que "l'auteur est toujours le personnage principal de son livre » («Vous devriez raconter»). La propension même à ne pas faire d'entretiens en personne, mais plutôt par courriel, n'est non pas—ou non seulement-un choix pratique, mais aussi esthétique : il se permet ainsi de maintenir une version fictionnelle de lui-même dans la réalité qui ne peut que renforcer cette même ambiguïté dans ses fictions. L'œuvre chevillardienne témoigne ainsi d'une convergence des instances auctoriales de Maingueneau, « une structure de nœud borroméen, dont les trois anneaux s'entrelacent de telle façon que, si l'on rompt l'un des trois, les deux autres se séparent. [...] Mais aucune de ces instances n'est 
isolable ou réductible aux autres, leur écart est la condition de la mise en mouvement du processus de création » (110). À travers chaque instance, les deux autres s'exposent et s'expriment. Ce nœud devient donc l'enjeu du projet d'écrivain chevillardien : les figures se convergent et se complètent mutuellement pour forger un métadiscours sur le statut de la littérature. Vivre de sa fiction et vivre dans sa fiction sont, dès lors, quasi synonymes.

\section{Les recettes des auteurs sont indigestes}

Les notes en bas de page de L'auteur et moi constituent donc autant un récit en soi qu'un prolongement logique des projets numériques et romanesques de l'auteur, un projet réalisé par une mise en dialogue embrouillée de l'auteurpersonne, l'écrivain et l'inscripteur des textes matériels et numériques. Les notes forment un récit non pas moins fictionnel que le récit qui raconte les échanges entre le narrateur-auteur et une femme dans un restaurant au sujet d'un gratin de chou-fleur. Les notes dépassent les frontières textuelles qui délimitent le genre romanesque en insérant au sein de la fiction des réflexions critiques situées normalement dans la réalité. Et pourtant, cette réalité ne peut qu'être fictionnelle puisque celui qui écrit les notes l'avoue lui-même dans le numéro 26 : « Pourquoi, en effet, serait-il interdit d'écrire un roman en bas de page ? Tant qu'il s'agit de papier, l'auteur, n'a-t-il pas pour vocation de le noircir ? "(115). Une question qui peut se comprendre dans deux sens. Premièrement, il s'agit d'un avertissement au lecteur puisque ce qui suit ce préambule est une nouvelle histoire qui prend forme dans cette même note. Le récit s'intitule La fourmi et occupe plus de 105 pages du récit, soit plus d'un tiers de L'auteur et moi. Deuxièmement, elle est également révélatrice des enjeux d'un processus créateur qui tâche d'assouplir les limites du romanesque. Les notes servent ainsi à résumer avec une grande pertinence le style de l'auteur et à examiner les possibilités du roman ; une réflexion nécessaire, selon Chevillard, face à l'insipidité littéraire de l'époque contemporaine.

La littérature « indigeste » fait l'objet d'une attaque régulière chez Chevillard, qui précise lors d'un entretien en 2005 :

[...] [i]l n'est pas très sain de céder à toutes les facilités de l'époque. [...] Un écrivain doit nécessairement être un peu réactionnaire, ce qui ne consiste évidemment pas à pleurer le bon vieux temps ou à demeurer figé dans des postures et des grammaires anciennes, mais à ne pas consentir avec complaisance à toutes les simplifications qui nous sont proposées pour faire de nous les joyeux consommateurs écervelés que le système aimerait bien voir se répandre. («Cheviller au corps »)

La réflexion de Chevillard rappelle celle de Dominique Viart et Bruno Vercier lorsqu'ils font référence à une littérature concertante (8), l'une des trois catégories qu'ils proposent pour décrire les tendances qui prévalent dans le milieu littéraire français depuis les années $1980^{5}$. Il s'agit d'une littérature symptomatique de notre temps, qui est, selon Viart et Vercier, " plus attentive à l'époque, à ses odes ou à ses humeurs ", mais selon " un autre registre, plus mondain et plus mercantile. [...] Nul doute que cette littérature traduise quelque chose de l'état social, mais elle ne le pense pas " (9). Cette catégorisation permet à Viart et Vercier de dépasser les préoccupations esthétiques pour souligner les enjeux d'ordre interprétatif et commercial de la littérature d'aujourd'hui. Dans cette perspective, " interprétation ", ou tout simplement " lecture », se rapproche de " consommation » et fait référence tant au marché de l'édition qu'à la circulation de la littérature, disons, "grand public ». Comme un repas de gratin de chou-fleur, cette littérature, fort critiquée par Chevillard, est marginalement comestible, mais sans valeur nutritive. C'est un plat prévisible, acceptable pour ceux qui ont tout simplement faim mais révoltant pour ceux qui s'attendent à plus de leur expérience « gastro-littéraire ». La littérature concertante met au premier plan la réponse à une demande du public, sans déstabiliser ses goûts.

II appert que la fausse simplicité de la littérature qui est une littérature concertante provient d'une complicité entre auteur et lecteur à laquelle s'attaque récemment Chevillard à plusieurs reprises dans une critique assez agressive du roman Les Souvenirs de David Foenkinos. Chevillard écrit à ce sujet :

De certains livres, nous pouvons dire encore avec faveur qu'ils sont de forme quadrangulaire parfaite, constitués d'un certain nombre de pages dûment numérotées selon la plus rigoureuse mathématique, après quoi notre enthousiasme retombe. [...] II relève d'un genre que j'appellerai le « roman de vérification ». (« Littérature pavillonnaire »)

Outre une critique de l'œuvre de Foenkinos, cet extrait révèle la critique plus large de ce que Chevillard nomme « le bon vieux roman » : " le roman qui consiste à raconter une histoire avec un début et une fin, ... [et qui] semble obéir au principe même de l'existence humaine. [...] Du coup on se confine dans l'usage d'une langue plate, très classique, un peu molle, bourrée inévitablement de clichés puisqu'on est amené à décrire des situations vécues par tous » ( "Cheviller au corps »). Le « bon vieux roman », le " roman de vérification » ou encore la " littérature concertante » se réduit à une recette : c'est une formule bien calculée, dont le résultat est prévisible et même réconfortant pour celui qui le consume. Pourtant, à la place du gratin dégoûtant, qui représente une grande partie de la littérature de 
nos jours, Chevillard voit un potentiel créateur. Dans un entretien publié quelques années après la parution de L'auteur et moi, Chevillard explique que :

La littérature est revêche, importune, outrecuidante. Ce ne sont pas des qualités bien reluisantes, mais ce sont les siennes. Il faut accepter en tout cas cet abord difficile, débrouiller en soi le fil de l'illisibilité parfois, et alors s'ouvre un monde de délices... amandes douces, lait d'ânesse, fontaines de miel... (« Je déteste la littérature »)

À cela s'ajoute la pensée d'Alexandre Gefen, qui, en citant la réflexion d'Arno Bertina dans « L'histoire et moi », souligne qu'à l'ère des réseaux numériques "la littérature doit, pour "se mesurer à la multiplicité du réel", devenir esthétiquement et génériquement "profane" et profanatrice, "impropre" et déstabilisante, "hétérodoxe et hétérogène" » (Gefen). Le flux presque perpétuel de textes numériques contribue à la multiplicité et à l'hétérogénéité de la littérature contemporaine et souligne également la critique de Chevillard. La difficulté potentielle de gérer et de digérer l'ampleur de la présence virtuelle aujourd'hui et de situer le rôle du lecteur par rapport à cette déstabilisation générique et esthétique est un obstacle important à considérer. Le monde virtuel, fait-il de la littérature un « monde de délice " ou sommes-nous, comme le suggère le protagoniste de L'auteur et moi, face à un monde de gratin de chou-fleur où " tout est dedans disloqué, rompu, moulu, réduit à l'état de débris, de déchets, d'immondices " (94) ?

Chevillard explique lors d'un entretien de 2011 qui s'intitule "L'autre personnage du livre, c'est le lecteur », que son projet n'est pas une attaque contre la littérature ou contre le roman, mais qu'il

[...] contrecarre plutôt les tentatives d'infiltration dans [s]a prose candide des genres littéraires constitués, si solidement institués même et aguerris par des siècles de pratique et d'illustration qu'ils s'imposent d'abord à [lui] aussi comme seules solutions possibles. Alors [il] [s]e braque, [il] résiste, [il] retourne contre elles-mêmes les puissances structurantes qui se font jour, [il] les dévoie, [il] essaie de gripper un peu les mécanismes de la machine à produire des livres. ("L'autre personnage du livre »)

Ce qui découle de ses efforts est une œuvre qui écrit le présent en même temps qu'il le critique. Voulant prendre sa distance avec les expérimentations formelles des avant-gardes, Chevillard, comme d'autres romanciers contemporains, ne refuse pas pour autant la tradition, d'où ce désir renouvelé de raconter les aventures de personnages qui invitent à une réflexion sur notre monde actuel. Aussi peut-on dire qu'une complicité est née entre tradition et expérimentation, qui ne nie pas le passé comme seul moyen de dire le présent et qui dépend même des outils romanesques ainsi que des partis-pris littéraires antérieurs pour accomplir son projet. En déplaçant le romanesque du côté de la rupture vers celui de l'accumulation, les détours digressifs, ludiques et parfois grinçants, qui saccadent et complètent l'œuvre chevillardienne, révèlent l'enjeu d'un projet d'écrivain qui ne se contente pas des attentes que l'étiquette de « romancier » lui impose. En tant que processus de déplacement, l'acte créateur s'étend au-delà des couvertures du roman, s'immisçant dans les notes de L'auteur et moi, aussi bien que dans son blog, ses entretiens, ses comptes rendus et sur son site-web.

La question qui s'ensuit est de savoir si le lecteur contemporain est nourri de force ou si ce qui le sustente revient à un choix personnel. D'une certaine manière, Chevillard critique un marché littéraire saturé de romans médiocres qui transforment les lecteurs en agents passifs : le type de lecteur qui ne s'appuie que sur « START » sur le four à microondes pour réchauffer son repas à emporter de la nuit précédente. Pourtant, dans un deuxième temps, les attaques systématiques contre le lecteur peuvent se comprendre comme appel à l'action et il semble que la réflexion de Chevillard va de plus en plus explicitement dans ce sens. II se demande si la littérature ne devrait pas plutôt « transforme[r] ce matériau élémentaire de la vie commune en expérience de conscience originale » ("Littérature pavillonnaire $»)$.

L'idée de « conscience originale » revient régulièrement dans les entretiens les plus récents de l'auteur. II s'agit de moments vécus par un lecteur qui ne se contente pas de l'idéal traditionnel auquel il était tenu. Ces expériences sont caractéristiques d'un lecteur critique qui préfère se soumettre à une situation désagréable plutôt que se tourner vers celle qui le réconforte. Il est celui qui répond instantanément à sa lecture, non pas avec un mépris imprudent, mais avec une ouverture d'esprit et une souplesse qui permettent à tout moment une transformation de perspective. Plutôt que d'être nourri de force par une littérature qui est le miroir de son temps, le lecteur est encouragé à expérimenter dans le but de découvrir de nouveaux plats.

Afin d'inciter une expérimentation littéraire et interprétative, Chevillard propose une coopération entre les instances auctoriales, le narrateur, le personnage et le lecteur comme prolongement logique de l'indistinction qu'il forge entre auteur-personne et auteur-persona. Lors d'un entretien avec Roger-Michel Allemand, Chevillard explique que : 
[...] l'auteur, le narrateur, le personnage, le lecteur sont dans mes romans des entités fictives, presque interchangeables, ce qui peut créer en effet une sensation d'inconfort, mais engage chacun dans l'aventure du livre [...]. Tout cela est d'autant plus nécessaire, si l'on veut que quelque chose se passe qui ne soit pas simple divertissement [...]. (153)

Dans la mesure où l'auteur, le narrateur, le personnage et le lecteur sont « presque interchangeables », l'image d'un lecteur qui s'installe agréablement dans son monde extralittéraire est devenue chose du passé. II est constamment dérangé par le texte, parfois sous forme de harcèlement, mais également par admiration pour son potentiel. Chevillard dit lui-même que «[I]e lecteur selon mon goût ne se laisse évidemment pas tout à fait prendre à mes leurres. Au moins je ne le considère pas comme Pavlov son chien " ( Douze questions »), soulignant l'indépendance et le libre choix interprétatif du lecteur. En somme, Chevillard écrit pour le lecteur qui apprécie la subtilité d'une truite aux amandes.

\section{Vers une tentative de goûts plus raffinés}

La critique de Chevillard développée dans son œuvre n'est pas un geste rhétorique, mais est constitutive de son esthétique. Au-delà d'une simple répugnance pour les recettes anciennes ou les dîners fastfood, Chevillard se demande comment prendre les mêmes ingrédients et créer quelque chose de susceptible d'inspirer l'expérimentation chez les écrivains et d'exploiter les goûts plus raffinés des lecteurs. En somme, comment mieux gérer la littérature afin de mieux la digérer ? La réponse à la question demeure peut-être dans l'impossibilité de réduire la réponse à un seul constat. La réflexion créative et interprétative qui parcourt les textes matériels et numériques, le paratexte et le péritexte, forment l'œuvre d'Éric Chevillard et pointent vers un refus de réduire la littérature à un tout homogène. Elle brouille toute distinction possible entre l'auteur-personne et l'auteur-persona, une confusion qui se forge dans les deux sens: en fin de compte les personnages chevillardiens deviennent-ils des extensions de lui-même ou bien Chevillard devient-il une extension de ses personnages ? La bidirectionnalité du propos semble être précisément l'objectif de l'auteur, qui ne privilégie aucun discours - que ce soit analogue, virtuel, réel ou fictionnel - au-delà d'un autre.

Selon Louise Merzeau, dans le contexte précis de la «traçabilité » à l'ère numérique, il s'agit d'une identité hétérogène façonnée par une " présence informationnelle » : " II n'y a plus d'un côté une identité stable (à protéger ou à exhiber) et de l'autre des données qui circulent, mais le façonnage réciproque et continu d'une présence informationnelle. [...] [L]es contours de l'identité sont eux-mêmes modifiés par le flux de données » (31). Dans un contexte littéraire, le projet transmédiatique vient compléter le métadiscours sur la littérature au cœur de l'œuvre chevillardienne et tout porte à croire que la dématérialisation du texte est propice à la rematérisalisation de l'auteur, sous une forme plus ouverte au dialogue, mais non moins sérieuse dans son propos.

La présence numérique n'empoisonne pas la présence matérielle, mais ajoute encore une autre dimension discursive. Ces discours se déroulent en concert avec le discours narratif et les instances auctoriales forgées, comme le suggère Maingueneau, créent une hybridité qui jette un défi aux structures narratologiques par lesquelles nous avons tendance, par convention, à appréhender la littérature. « Auteur-papier » et « auteur-numérique » ne sont plus des oppositions binaires suffisantes pour tenir compte de l'identité auctoriale ou de celle du roman. L'hétérogénéité du texte et de l'auteur forge des discours qui s'entrecroisent, se mêlent, s'informent et s'enrichissent mutuellement. Comme la tension créée entre auteur, narrateur, personnages et lecteurs par le biais d'un processus créateur qui devient topos de l'œuvre chevillardienne, il n'existe pas de hiérarchisation du discours selon une frontière fiction/réalité, genre fictionnel/genre dénotatif, texte matériel/texte virtuel, mais une ouverture de l'acte créateur vers de nouvelles possibilités romanesques.

\section{Notes}

${ }^{1}$ Nous rappelons que dans son essai « La mort de l'auteur » (1967), Roland Barthes écrit : « Donner un Auteur à un texte, c'est imposer à ce texte un cran d'arrêt, c'est le pourvoir [ou pouvoir ?] d'un signifié dernier, c'est fermer l'écriture. Cette conception convient très bien à la critique, qui veut alors se donner pour tâche importante de découvrir l'Auteur (ou ses hypostases: la société, l'histoire, la psyché, la liberté) sous l'œuvre : l'Auteur trouvé, le texte est "expliqué", le critique a vaincu [...]. [L]a littérature (il vaudrait mieux dire désormais l'écriture) en refusant d'assigner au texte (et au monde comme texte) un " secret », c'est-à-dire un sens ultime, libère une activité que l'on pourrait appeler contre-théologique, proprement révolutionnaire [...]. Pour rendre à l'écriture son avenir, il faut en renverser le mythe : la naissance du lecteur doit se payer de la mort de l'auteur » (68-69). 
${ }^{2}$ Proposé par Wolf Schmid en 1973 dans Der Textaufbau in den Erzählungen Dostoevskijs et remanié dans son ouvrage Narratology : An Introduction publié en 2000, le schéma narratologique illustre bien les relations d'opposition entre fiction et réalité et instaure des niveaux de fictionnalité tout en soulignant le rapport binaire à l'intérieur de chacun : I'Auteur abstrait et le Lecteur abstrait; le Narrateur et le Narrataire ; les divers personnages en dialogue dans le récit.

${ }^{3}$ Nous pensons notamment aux travaux de Hans Robert Jauss et de Wolfgang Iser, en plus de ceux d'autres théoriciens comme Umberto Eco, Stanley Fish, Wayne Booth et Gérard Genette.

${ }^{4}$ Crab est le protagoniste récurrent de La nébuleuse de crab et Un fantôme, et Albert Moindre est le protagoniste de Sans l'orang outan, L'œuvre posthume de Thomas Pilaster et Dino Egger.

${ }^{5}$ Dominique Viart et Bruno Vercier comparent ici la littérature concertante à la littérature consentante. Cette dernière regroupe les ouvrages destinés à être vendus au grand public et qui se rapprochent du pur divertissement. À ces deux catégories s'ajoute une troisième, la littérature déconcertante, qui ne cherche à pas à correspondre aux attentes du lectorat, mais contribue à les décaler afin d'inciter la réflexion critique. Cf. La littérature française au présent : héritage, modernité, mutations.

\section{Bibliographie}

Barthes, Roland. « Introduction à l'analyse structurale des récits. » Communications 8 (1966) : 1-27. Imprimé.

. «La mort de l'auteur. » Le bruissement de la langue : essais critiques IV, Paris : Seuil, 1984. Imprimé.

Bon, François. Après le livre. Paris : Seuil, 2011. Imprimé.

Chevillard, Éric. L’auteur et moi. Paris : Éditions de Minuit, 2012. Imprimé.

. L'Autofictif. Web. 15 novembre 2015.

. "L'autre personnage du livre, c'est le lecteur. » Entretien avec Aline Girard, Luc Ruiz et Alain Schaffner, Centre d'études du roman et du romanesque (22 septembre 2006). Web. 13 novembre 2015

—_ « Biographie » Éric Chevillard. Site web professionnel. Web. $1^{\text {er }}$ novembre 2015.

. «Cheviller au corps. » Entretien avec Emmanuel Favre, Le Matricule des anges 61 (mars 2005). Web. 17 novembre 2015.

. "Choir "sans intention" - mais vers le haut. » Entretien avec Roger-Michel Allemand, @nalyses : Revue de critique et de théorie littéraire 5.1 (2010) : 149-62. Web. 14 novembre 2015.

. «Des leurres ou des hommes de paille. » Entretien avec Pascal Riendeau, Roman 20-50 46 (2008) : 11-22. Imprimé.

_. « Douze questions à Éric Chevillard. » Entretien avec Florine Leplâtre, Inventaire/Invention (novembre 2006). Web. 28 novembre 2015.

—. "Je déteste la littérature qui ne sublime rien. » Entretien avec Matthieu Giroux, Ragemag 3 (février 2013). Web. ${ }^{\text {er }}$ novembre 2015. 
Shaw, Aimie. «Vivre de sa fiction, vivre dans sa fiction : le processus créateur au défi du numérique. » Nouvelle Revue Synergies Canada, Nº (2017)

_. « Littérature pavillonnaire. » Le Monde des livres (25 août 2011). Web. $1^{\mathrm{er}}$ novembre 2015.

—. " Vous devriez raconter une histoire que tout le monde connaît déjà. " Entretien avec Nicolas Vives, Pages des libraires 85 (2003). Web. 17 novembre 2015.

Držková, Kateřina. « Éric Chevillard, un représentant du contemporain aux Éditions de Minuit. » Sborník Prací Filozofické Fakulty Brnenské Univerzity Studia Minora Facultatis Philosophicae Universitatis Brunensis 24 (2003) : 69-76. Imprimé.

Gefen, Alexandre. « Le devenir numérique de la littérature française. » Implications Philosophiques (19 juin 2012). Web. 26 novembre 2015.

—_. « L'histoire et moi. » Inculte 11 (septembre 2006). Web. 28 novembre 2015.

Maingueneau, Dominique. Le discours littéraire : paratopie et scène d'énonciation. Paris : Armand Colin, 2004. Imprimé.

Merzeau, Louise. "Présence : de la gestion d'une identité à l'exercice d'une liberté. » Documentaliste : Sciences de l'information 47.1 (2010) : 28-33. Web. 28 novembre 2015.

Ruiz, Ugo. « Ethos et blog d'écrivain : le cas de L'Autofictif d'Éric Chevillard » COnTEXTES 13 (2013). Web. 13 novembre 2015.

Viart, Dominique et Bruno Vercier. La littérature française au présent : héritage, modernité, mutations. Paris : Bordas, 2008. Imprimé. 